\title{
The Role of Mixotrophy in Southern Ocean Ecosystems
}

\section{The Sensitivity of Model Dynamics to the Magnitude and Form of Mixotroph Interactions between Plankton}

\author{
John Norbury • Irene M. Moroz • Roger \\ Cropp
}

Received: date / Accepted: date

\begin{abstract}
We investigate the influence of mixotrophy on the dynamical properties of a six population model of a three trophic level Southern Ocean ecosystem. We find that including mixotrophic interactions between the lowest trophic level populations can significantly influence the dynamics of the highest trophic level populations, and in extreme cases lead to extinctions. Significantly, not only is the strength of the mixotrophic interaction important, it matters how it is included in the model, as a specialist or generalist grazer. We note in particular that the generalist formulation is inappropriate for "green" mixotrophs that fuel the majority of their growth by photosynthesis. The model can have complicated dynamics when subject to large amplitude, regular forcing, suggesting the sea ice - salps link may be obfuscated by endogenous population oscillations. Further, we observe that constructing the model within the Conservative Normal framework allows insights into the bifurcation behaviour of the model.
\end{abstract}

J. Norbury

Mathematical Institute

University of Oxford

Andrew Wiles Building, ROQ

Oxford OX2 6GG, UK

E-mail: john.norbury@lincoln.ox.ac.uk

I. M. Moroz

Mathematical Institute

University of Oxford

Andrew Wiles Building, ROQ

Oxford OX2 6GG, UK

E-mail: Irene.Moroz@maths.ox.ac.uk

R. Cropp

Faculty of Environmental Sciences

Griffith University

Gold Coast, 4216

Queensland, Australia

E-mail: r.cropp@griffith.edu.au 
Keywords Mixotrophy · Chaos · Specialist - Generalist · Plankton · Environmental Forcings

\section{Introduction}

The Southern Ocean is one of the world's major marine systems, supporting iconic species such as krill and whales, and ecosystems that may influence the Earth's future climate. The ecosystem services provided by the Southern Ocean range from providing fisheries products (krill landings are around 11\% of global fisheries production) to the cultural and aesthetic benefits of iconic regions and biota, to climate regulation [20]. Antarctic plankton ecosystems may influence climate through their production of dimethylsulphide and consumption of carbon dioxide [52]. While processes at the bottom of the Southern Ocean food chain may directly affect climate, ecosystems function as integrated systems, with bottom-up processes in which lower level interactions, for example among plankton, affect higher trophic levels such as whales; and top-down processes where, for example, harvesting of whales may affect plankton populations. Southern Ocean living resources have long been exploited, with sealing and whaling active since the late 18th century. The need for management of these resources has often focussed on the role of krill, which is a key prey species for many Southern Ocean predators [3,24,29]. Some of the early work on the consequences of harvesting living resources expounded the need for ecosystem approaches to understand the impacts of Southern Ocean fisheries on krill populations (for example, May et al., [32]). Since the early work of May et al. [32], over 40 models of the impact of harvesting of krill populations on Southern Ocean ecosystems have been published [22]. However, it has been relatively recently recognised that a fundamental trophic interaction is missing from these models.

A key distinction that has formed the basis of most modelling of marine ecosystems is the separation of the plankton into two distinct groups: phytoplankton (autotrophic plants) and zooplankton (heterotrophic animals). Recent research suggests that this approach may not represent the true trophic behaviour of these populations [18]. Flynn et al. [18] estimate that approximately $80 \%$ of plankton are mixotrophs, that can simultaneously function as both autotrophs (that photosynthesise) and heterotrophs (that consume other organisms). Much of the work on mixotrophy has focussed on the physiological level, representing these processes in detail $[17,12,53,37,38]$. The complexity of these models can then be overwhelming in the context of inferring the major processes driving the properties and their sensitivity to parameter values, many of which are poorly constrained by data. Such models often produce interesting ecosystem dynamics, ranging from stabilisation [38] to destabilisation [12] of ecosystems. The detail of the biophysical processes in these models can obfuscate the population-level processes that determine the properties of the ecosystems that is of fundamental importance to the question of whether the 
inclusion of mixotrophic interactions in plankton will fundamentally change the dynamics of marine ecosystems.

The efficacy of mixotrophy as a feeding strategy for populations has been demonstrated theoretically by Cropp and Norbury [6], but the potential for such a fundamental low trophic level distinction to affect the dynamics of populations in the Southern Ocean, from plankton to whales, remains largely unexplored. Increasing evidence that population interactions such as mixotrophy dominate interactions in marine plankton ecosystems [18] points to a need to develop a robust theoretical basis to understand how these interactions influence the dynamics of food webs. Zooplankton are known to adopt different foraging strategies such as prey selection [43], diurnal vertical migration [49], and even feeding expeditions to abyssal depths [4]. Marine planktonic omnivores, in particular mixotrophs, may also change their feeding preferences in response to changes in temperature [54]. Changes in trophic interactions at the base of a food web may have significant implications for the dynamics of the whole system. For example, Jost et al. [26] found that the inclusion of mixotrophy between autotrophs using a Lotka-Volterra predation term could stabilise an ecosystem model.

The constraints placed on developing complex models of Southern Ocean ecosystems are many, with a paucity of data on parameter values being only one of a number of knowledge gaps identified [41]. The choice of the functional form used to represent interactions between populations in ecosystems is an ongoing point of discussion among ecological modellers [40]. The theoretical bases for many of the simple two-population interaction terms were set out early, for example by Holling [23], Ivlev [25], Lotka [31], and Volterra [51], but there remains little theoretical or empirical evidence to justify a choice of one form over another. A number of studies have noted that subtle changes to even simple functional forms may have important influences on properties of the systems $[8,19,35,36]$. The choices made for these functional forms can have profound influences on system properties. For example, Cropp and Norbury [10] showed that for a general class of models it was possible to ensure that no population ever went extinct in a model of any complexity by choosing functional forms to have certain simply identified properties.

There is however, some consistency among processes involved in mixotrophy that facilitate the inclusion of mixotrophy in plankton models with sufficient robustness to justify a first examination of how the inclusion of this process might affect the properties of the whole ecosystem. The functional form describing substrate-limited enzymatic processes [34], the growth of phytoplankton on nutrients [13] and the grazing of zooplankton on phytoplankton [23] are all hyperbolic functions that saturate as resource availability increases. Even though the physiological processes involved in a mixotroph functioning in autotroph mode, taking up inorganic nutrient to fuel photosynthesis, are very different to the physiological processes involved in a mixotroph functioning in heterotroph mode grazing on a prey, the same functional form may be used to describe both processes. This facilitates examining how mixotrophs re- 
spond to varying levels of resources associated with autotroph and heterotroph behaviours.

In this paper we consider the simplest case of how the inclusion of a mixotrophic interaction at the lowest trophic level (one primary producer "grazing" upon the other) influences the behaviour of the system and examine the importance of the foraging strategies used by mixotrophic plankton. We consider two forms of mixotrophic grazing: specialist, where the mixotroph preferentially forages for a particular prey; or generalist, where the mixotroph consumes prey whenever it is encountered $[8,27,50]$. While a reasonable approach for modelling different grazing strategies by heterotrophs, the validity of this approach for mixotrophs is yet to be determined, and it should be considered a first approximation based on a mathematical perspective rather than biological verisimilitude. This point is revisited in the Discussion.

We find that regularly oscillating coexisting population states can be destabilised by increasing mixotrophy, generating irregular oscillations of plankton and ice algae (even though the seasonal oscillation of the annual sea ice coverage is regular), and even to extinctions of key populations such as krill and whales. A key result for ecologists is that subtle variations in trophic interactions at the lowest trophic level can produce dramatic changes in the population dynamics of higher trophic level populations, suggesting that resolving the ubiquity, form and strength of mixotrophic (and more generally omnivorous, see [39]) interactions among plankton may be a fundamental pre-requisite to being able to effectively manage iconic megafauna populations in Antarctica. An interesting technical result for ecosystem modellers is that the inclusion of mixotrophy for phytoplankton in the commonly-used generalist grazing form leads to the extinction of phytoplankton until the level of mixotrophic grazing increases sufficiently to offset the spurious reduction in photosynthesis that results from the generalist form.

\section{The Southern Ocean Ecosystem Model (SOEM)}

We investigate a canonical model of the Southern Ocean near-shore ecosystem (the SOEM, Fig. 1) in which pelagic phytoplankton $(P)$ are distributed throughout the water column and ice algae $(A)$, that grow attached to the underside of sea ice, form the base of the food web. They compete for dissolved inorganic nitrogen $(N)$ to fuel their growth, but the ice algae further rely on the extent of the sea ice cover to provide a substrate to grow on. The pelagic phytoplankton are mixotrophs that may also consume the ice algae, but the ice algae lack this ability in the model in recognition that ice algae are mostly diatoms that do not engage in phagotrophy. Krill $(K)$ and salps $(S)$ constitute the grazer level, with the krill competing with the salps to feed on the phytoplankton, but krill are the sole grazer of the ice algae. The highest trophic level of the ecosystem comprises whales $(W$, notionally representing seasonal feeders on krill) and fish ( $F$, notionally representing year-round feed- 
ers on krill). The whales feed only on the krill, but the fish feed on both the krill and the salps.

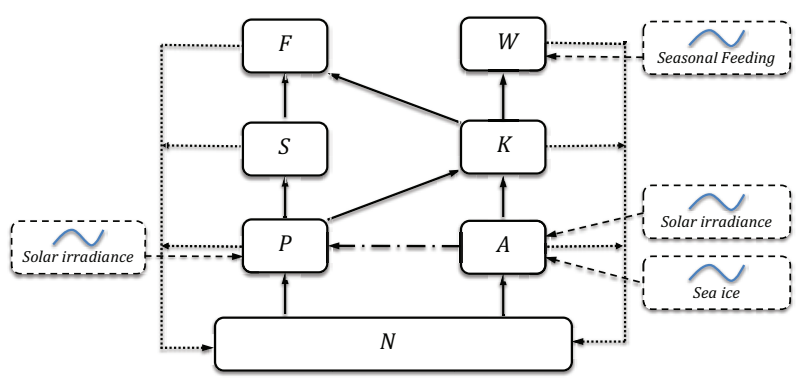

Fig. 1 Food web diagram for the SOEM. Solid lines show movement of mass of limiting nutrient due to consumption of inorganic nutrients $(A$ and $P$ ) or consumption of other organisms $(S, K, F$, and $W$ ). Dotted lines show movement of mass due to recycling of wastes, respiration, and sloppy feeding. (Note that remineralisation of organic nutrient is implicit and is assumed to be very fast compared to the time scales of the other organisms.) The dashed-dotted line shows the mixotrophy of $P$ feeding on $A$ in addition to its usual growth on inorganic nutrient by photosynthesis. Environmental forcings may act upon $A$ and $P$, and $W$ may be migratory.

The structure and components of foodwebs such as Fig. 1 are very similar to that proposed by May et al. [32] as suitable for generic model investigations of the impact of fisheries on Antarctic ecosystems. We also abstract the species involved into very broad trophic guilds intended to capture the major feeding interactions. The "whales" do not therefore represent a specific species of whale, but in fact all populations that seasonally feed on krill in Antarctica; similarly, the "fish" represent all populations that permanently feed in krill (i.e. resident populations). The populations we model should not be considered to represent any particular species, and therefore cannot be attributed with any specific traits. The model is deliberately abstracted and simplified in order that key attributes of the trophic interactions may be discerned. For a detailed model of an Antarctic ecosystem, that also informed the development of this model, the reader is referred to Bates et al. [2].

The equations describing these interactions, that determine how the ecosystem changes over time and define the SOEM, are detailed in equation (1)(a-f). The modelling framework underpinning these equations, and the derivations of the interaction terms are explained in detail in [6], [8], and [11]. 


$$
\begin{aligned}
& \dot{A}=A {\left[\frac{\mu_{A} R_{A} N}{N+\kappa_{A}}-\frac{\phi_{A P} P}{A+\rho N+\kappa_{A P}}-\frac{\phi_{A K} K}{A+P+\kappa_{K}}-\sigma_{A}\right], } \\
& \dot{P}=P\left[\frac{\mu_{P} R_{P} N}{N+\rho A+\kappa_{A P}}+\frac{\phi_{A P} A}{A+\rho N+\kappa_{A P}}-\frac{\phi_{P K} K}{A+P+\kappa_{K}}\right. \\
&\left.\quad-\frac{\phi_{P S} S}{P+\kappa_{S}}-\sigma_{P}\right], \\
& \dot{K}=K\left[\frac{\phi_{A K}\left(1-\psi_{A K}\right) A+\phi_{P K}\left(1-\psi_{P K}\right) P}{A+P+\kappa_{K}}-\frac{\phi_{F K} F}{K+S+\kappa_{F}}\right. \\
& \dot{S}=S\left[\frac{\phi_{P S}\left(1-\psi_{P S}\right) P}{P+\kappa_{S}}-\frac{\phi_{S F} F}{K+S+\kappa_{W}}-\sigma_{S}\right], \\
& \dot{F}=F\left[\frac{\phi_{K F}\left(1-\psi_{K F}\right) K+\phi_{S F}\left(1-\psi_{S F}\right) S}{K+S+\kappa_{F}}-\sigma_{F}-\epsilon_{F} F\right], \\
& \dot{W}=W\left[\frac{\phi_{K W}\left(1-\psi_{K W}\right) R_{W} K}{K+\kappa_{W}}-\sigma_{W}-\epsilon_{W} W\right] .
\end{aligned}
$$

We measure the biomass in each population in terms of the amount of the key limiting macronutrient it contains, in this case, nitrogen. We determine the amount of dissolved inorganic nitrogen in the system $(N)$, and note that Southern Ocean ecosystems, in common with most other systems [50], recycle their limiting nutrient very efficiently. The total amount of limiting nutrient cycling in the food web remains constant, so that $A+P+K+S+F+W+N=$ $N_{T O T A L}$, or equivalently we have the equation that describes the dynamics of the nutrient pool:

$$
\dot{N}=-(\dot{A}+\dot{P}+\dot{K}+\dot{S}+\dot{F}+\dot{W}) .
$$

Equation (2) arises from conservation of the recycling limiting nutrient in the food web of Fig. 1.

Finally, we scale all the populations by the total amount of cycling limiting nutrient $N_{\text {TOTAL }}$, so that each population biomass is expressed in terms of the fraction of the total resources in the system that it has sequestered, and $A+P+K+S+F+W+N=1$. The various parameters appearing in equations (1)(a-f) are defined in Table 1, together with typical parameter values, as estimated via the SeBEDes method [7]. This parameterisation method is based on the Conservative Normal (CN) modelling framework [6], which has been used as the theoretical basis to develop the SOEM. In addition to facilitating this parameterisation approach, the $\mathrm{CN}$ framework has the added benefit that the stability of the "boundary" eigenvalues of the system, that control the extinctions of populations, are easily determined from the sign of the per capita growth rate at the boundary where the population vanishes. This attribute facilitates understanding of the processes driving some of the bifurcation behaviour that we see in the SOEM, which include equilibrium 
solution states changing to either chaotic dynamics or extinctions as certain key parameters vary.

\subsection{Grazing}

Grazing in marine ecosystem models is commonly based on the hyperbolic saturating form commonly referred to as Holling Type II after Holling's [23] derivation of the grazing disc equation. This is often modified for omnivorous grazers, that feed on multiple prey. Omnivores may use one of two strategies when foraging for prey: they may have prey-specific foraging times, commonly referred to as specialist foragers; or they may have a common foraging time across all prey [27]. Many ecosystem models that include marine plankton represent grazing interactions between omnivorous populations and their prey using a generalist functional form [44], and the derivations of these forms are well-documented $[47,40]$. Specialist grazing is less-often used in plankton models, perhaps because it can produce "over-grazing" if resources are not rigorously accounted for.

Koen-Alonzo [27] provides a detailed theoretical justification for the formulation of specialist grazing terms, and Cropp et al. [8] extended this derivation to provide the transitional functional forms used in equations 1 (a) and 1(b). They also showed that when implemented in a resource-based system the choice of specialist or generalist foraging strategies for zooplankton could have significant implications for the dynamics of plankton ecosystem models.

We utilise the omnivore grazing formulation to describe the different strategies available to the mixotroph in the SOEM model. As autotrophy and heterotrophy involve different cellular metabolic processes, this raises the question of whether it is reasonable to assume that the availability of prey will affect a cell's autotrophic processes, and vice versa. We follow the approach of Ward et al. [53] and assume that the surface of a mixotrophic pelagic phytoplankton cell is comprised of handling sites that are specialised for autotrophy (i.e. the uptake of nitrogen) and sites that are specialised for heterotrophy (i.e. the cross-membrane transport of prey). Two cases of the distribution of these sites on the surface of a cell require consideration: either the sites are clustered (for example, one hemisphere is composed entirely of autotrophy sites and one entirely of heterotrophy sites) so that the processes of autotrophy and heterotrophy effectively do not impede each other; or the sites are intermingled so that they do. We assume the latter, so that in the absence of prey the autotrophy sites are active, but as the availability of prey increases, more heterotrophy sites activate and this results in the intermingled autotrophy sites becoming unavailable for photosynthesis. Our mixotroph process formulation functions so that an increase in prey availability reduces photosynthetic activity, and a reduction in prey availability produces an increase in photosynthesis.

We allow both specialist and generalist grazing within the same model by including a transition parameter, $\rho$ in the equations for $A$ and $P$ [8]. This allows us to transition smoothly and continuously between specialist mixotro- 
phy $(\rho=0)$ and generalist mixotrophy $(\rho=1)$. Although $\rho=0$ and $\rho=1$ are the only values of $\rho$ with clear ecological definitions, the mathematics allows us also to consider the transition values $0<\rho<1$. The use of the same functional form for both nutrient uptake in the autotrophic component, and prey consumption in the heterotrophic component of the mixotroph's feeding does not require that these occur through the same physiological processes. Although autotroph uptake of nutrient and heterotroph consumption of prey occur through fundamentally different physiological processes, at the population level, resource limitation functions in the same way irrespective of physiology - that is, the Michalis-Menten substrate limited uptake function has the same form as the Droop quota-limited growth function which has the same form as the Holling Type II grazing disc equation. The mixotroph functions in equations 1(a) and 1(b) are consistent with the physiological differences in uptake pathways.

The omnivores in the higher two trophic levels, krill and fish, are both generalist predators. The grazing interactions of these populations are not a particular focus of investigation in this paper, so we have used a generalist Holling Type II form for all of these interactions [47] . We parameterised these grazing functions with moderate to large half-saturation coefficients so that the populations are mostly operating in the linear phase of their grazing and the difference between Holling Type I and II functional forms is minimised.

\subsection{Seasonal Forcing}

Seasonal environmental forcings are included in the model as ice algae and phytoplankton growth modifiers $R_{A}$ and $R_{P}$, which incorporate the dominant environmental forcings controlling primary productivity in the Southern Ocean: solar irradiance and sea ice extent. We include seasonally varying whale interactions (Fig. 1) that occur due to migrations of whales (and other consumers of krill such as migratory seabirds) into and out of Antarctic waters each year [5].

The forcing data for both ice algae and phytoplankton were modelled by sine functions with means, amplitudes and relative phases chosen to reproduce climatological variations observed by the SeaWiFS and MODIS satellite sensors and recorded in the MERRA database available via NASA's Giovanni analysis tool (https://giovanni. gsfc.nasa.gov/giovanni/). We used NASA's Photosynthetically Available Radiation ( $P A R$, in units of Einstein $/ \mathrm{m}^{2} /$ day), a measure of daily average sea surface irradiance corrected for cloud cover, the surface ocean mixed layer depth ( $M L D$, in units of metres), and sea ice fraction $(I C E)$ for the period January 1979 to December 2015 for the region 
$90^{0}-180^{0} \mathrm{E}, 60^{0}-90^{\circ} \mathrm{S}$ to derive the climatological forcings:

$$
\begin{aligned}
& P A R=48+47 \sin \left(\frac{2 \pi(t+100)}{365}\right), \\
& M L D=58+12 \sin \left(\frac{2 \pi(t-50)}{365}\right), \\
& I C E=60+40 \sin \left(\frac{2 \pi(t-150)}{365}\right) .
\end{aligned}
$$

We used these data to estimate the average mixed layer irradiance experienced by the phytoplankton $\left(I_{A V}\right)$, where the light extinction coefficient $k_{L}=0.05$ per metre:

$$
I_{A V}=\frac{P A R}{k_{L} M L D}\left(1-e^{\left(-k_{L} M L D\right)}\right)
$$

We scaled the sea surface irradiance $(P A R)$ and the average mixed layer irradiance $\left(I_{A V}\right)$ by a term describing how light limitation influences phytoplankton growth [46]:

$$
I_{L I M}=I_{S A T} \sqrt{\left(1+\left(\frac{P A R}{I_{S A T}}\right)^{2}\right)},
$$

where the phytoplankton saturating irradiance $I_{S A T}=50$ Einstein $/ \mathrm{m}^{2} /$ day. Then the ice algae and phytoplankton environmental forcing functions are, respectively:

$$
R_{A}=\left(\frac{P A R}{I_{L I M}}\right) I C E, \text { and } \quad R_{P}=\frac{I_{A V}}{I_{L I M}} .
$$

The feeding function for whales is a smoothed square wave, generated by combining three piecewise sections of the error function:

$$
R_{W}= \begin{cases}1-0.5\left(1+\frac{2}{\sqrt{\pi}} \int_{0}^{0.05 *(t-60)} e^{-x^{2}} d x\right), & \text { if } 0 \leq t<160 \\ 0 & \text { if } 160 \leq t<205 \\ 0.5\left(1+\frac{2}{\sqrt{\pi}} \int_{0}^{0.05 *(t-305)} e^{-x^{2}} d x\right), & \text { if } 205 \leq t<365\end{cases}
$$

We scaled $R_{A}$ and $R_{P}$ by their maximum values so that the forcings varied between 0 and 1 . The forcings used in the analysis have realistic amplitudes and phase differences (Fig. 2). Note that we have scaled the rates in the model against a typical phytoplankton growth rate, however, as phytoplankton are commonly measured growing at a rate of 1 per day (see Table 1), we have used days for the time scales of the forcings. 

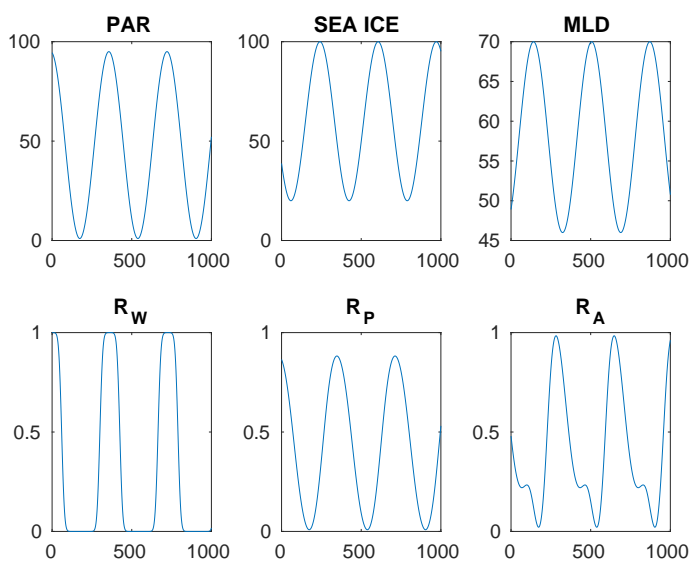

Fig. 2 Environmental forcings ( $P A R, I C E$ and $M L D)$, and the forcing functions $R_{W}$ $R_{P}$, and $R_{A}$ used for the SOEM food web model forced simulations. The $x$ axis has nondimensional time units where one unit is one day, assuming 365 days per year.
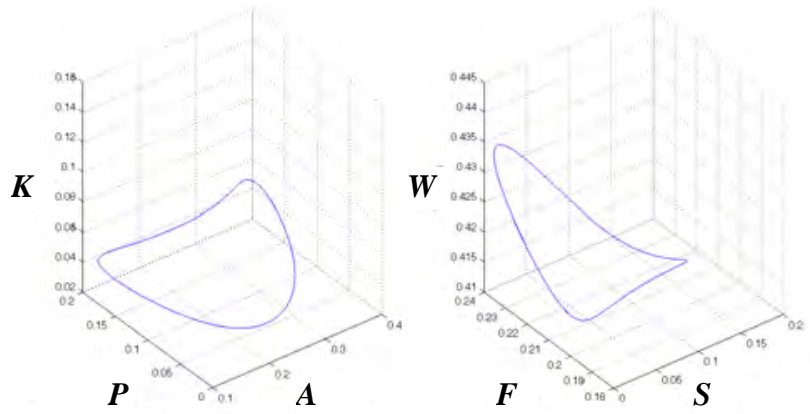

Fig. 3 The endogenous dynamics of the unforced equations (1) with $R_{A}=1=R_{P}=R_{W}$ using the parameter values in Table 1 . The system regularly cycles through a sequence of exactly repeating blooms of each population, as is evident in Fig. 4

\subsection{Dynamics}

The endogenous dynamics of the canonical unforced system (i.e. equations (1) with $R_{A}=1=R_{P}=R_{W}$ ) are a six-dimensional limit cycle, which is shown in phase diagram form in Fig. 3, and in time series form in Fig. 4.

The analysis we present in this paper focuses on how the inclusion of a mixotrophic interaction between the two primary producers, influences the dynamical properties of the SOEM. We consider different strength interactions, starting from low ("green") mixotrophy, where the mixotroph grows mostly by photosynthesis, and increasing until the system collapses, well short of the phytoplankton acting as a strong ("red") mixotroph, where the mixotroph 
Table 1 The definitions and default values of parameters used in (1). Default values were estimated using the SeBEDes method [7]

\begin{tabular}{|c|c|c|}
\hline Parameter & Role & Value* \\
\hline$\mu_{A}$ & Maximum growth rate of ice algae & 1.00 \\
\hline$\kappa_{A}$ & Ice algae half-saturation coefficient for nutrient uptake & 0.10 \\
\hline$\sigma_{A}$ & Ice algae mortality rate & 0.0001 \\
\hline$\mu_{P}$ & Maximum growth rate of phytoplankton & 1.0 \\
\hline$\kappa_{A P}$ & $\begin{array}{l}\text { Phytoplankton half-saturation constant for } N \text { uptake and } \\
\text { grazing onice algae }\end{array}$ & 0.10 \\
\hline$\phi_{A P}$ & Rate of phytoplankton grazing on ice algae & $0-0.25$ \\
\hline$\sigma_{P}$ & Phytoplankton mortality rate & 0.00005 \\
\hline$\phi_{A K}$ & Rate of krill grazing rate on ice algae & 0.50 \\
\hline$\psi_{A K}$ & $\begin{array}{l}\text { Fraction of ice algae, grazed by krill, lost to sloppy eating, } \\
\text { respiration and excretion }\end{array}$ & 0.25 \\
\hline$\phi_{P K}$ & Rate of krill grazing on phytoplankton & 0.25 \\
\hline$\psi_{P K}$ & $\begin{array}{l}\text { Fraction of phytoplankton, grazed by kKrill, lost to sloppy } \\
\text { eating, respiration and excretion }\end{array}$ & 0.25 \\
\hline$\kappa_{K}$ & $\begin{array}{l}\text { Half-saturation constant for krill, grazing on ice algae and phy- } \\
\text { toplankton }\end{array}$ & 0.20 \\
\hline$\sigma_{K}$ & Krill mortality rate & 0.10 \\
\hline$\phi_{P S}$ & Rate of salps grazing on phytoplankton & 0.33 \\
\hline$\psi_{P S}$ & $\begin{array}{l}\text { Fraction of phytoplankton, grazed by salps, lost to sloppy eat- } \\
\text { ing, respiration and excretion }\end{array}$ & 0.20 \\
\hline$\kappa_{S}$ & Half-saturation constant for salps, grazing on phytoplankton & 0.20 \\
\hline$\sigma_{S}$ & Salps mortality rate & 0.005 \\
\hline$\phi_{K F}$ & Rate of fish grazing on krill & 0.25 \\
\hline$\psi_{K F}$ & $\begin{array}{l}\text { Fraction of krill, grazed by fish, lost to sloppy eating, respira- } \\
\text { tion and excretion }\end{array}$ & 0.50 \\
\hline$\phi_{S F}$ & Rate of fish grazing on salps & 0.25 \\
\hline$\psi_{K F}$ & $\begin{array}{l}\text { Fraction of salps, grazed by fish, lost to sloppy eating, respi- } \\
\text { ration and excretion }\end{array}$ & 0.50 \\
\hline$\kappa_{F}$ & Half-saturation constant for fish, grazing on krill and salps & 0.90 \\
\hline$\sigma_{F}$ & Fish mortality rate & 0.01 \\
\hline$\phi_{K W}$ & Rate of whales grazing on krill & 0.05 \\
\hline$\psi_{K W}$ & $\begin{array}{l}\text { Fraction of krill, grazed by whales, lost to sloppy eating, res- } \\
\text { piration and excretion }\end{array}$ & 0.50 \\
\hline$\kappa_{W}$ & Half-saturation constant for whales, grazing on krill & 1.0 \\
\hline$\sigma_{W}$ & Whales mortality rate & 0.0014 \\
\hline$\epsilon_{F}$ & Fish closure coefficient & 0.02 \\
\hline$\epsilon_{W}$ & Whales closure coefficient & 0.001 \\
\hline
\end{tabular}

grows mostly by predation. We also consider different grazing strategies, specialist and generalist. Finally, we consider how the results we find from the idealised case transfer to the more realistic scenario where the populations are influenced by a seasonally changing environment. 
$\boldsymbol{A}$

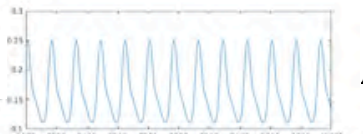

$\boldsymbol{K}$
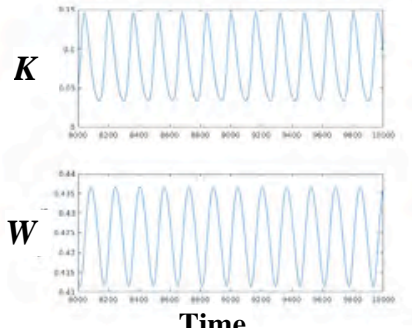

Time
$\boldsymbol{P}$

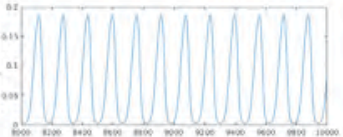

$S$

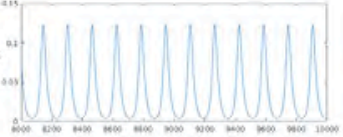

$\boldsymbol{F}$

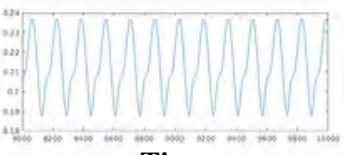

Time

Fig. 4 A time series depiction of the population dynamics of the unforced system equations (1) with $R_{A}=1=R_{P}=R_{W}$ using the parameter values in Table 1 . The system regularly cycles through a sequence of exactly repeating blooms of each population. This figure shows the same data as Fig. 3.

\section{Results}

We first consider the effect on the system of allowing $P$ to function as a specialist mixotroph grazer on inorganic nutrient $N$ and ice algae $A$ by increasing $\phi_{A P}$ to non-zero values and setting $\rho=0$ (Fig. 5). The dynamics of this mixotroph system evolve from the dynamics of the canonical unforced system, which are evident on the left axis of Fig. 5 where $\rho=0$. Here we see that the maximum value of the $P$ bloom observable in Fig. 3 agrees with the $P_{\max }$ value in Fig. 5 . As we increase $\phi_{A P}$ we retain the simple limit cycle with the amplitude of the $P$ bloom increasing smoothly until $\phi_{A P} \approx 0.02$. At this point, a second period is introduced into the limit cycle, and this double oscillation continues until $\phi_{A P} \approx 0.035$ when a sequence of period doubling initiates and chaotic dynamics are established at $\phi_{A P} \approx 0.0375$. The chaotic dynamics continue as $\phi_{A P}$ increases further, interspersed with the usual small windows of stability and limit cycles, until the ice algae, krill and whale populations go extinct at $\phi_{A P} \approx 0.067$. We are then left with the stable steady state $\left\{P^{*}=0.22, S^{*}=0.19, F^{*}=0.58\right\}$, that remains for all higher values of $\phi_{A P}$.

Of particular note in Fig. 5 is the very small value of $\phi_{A P}$ at which chaotic dynamics develop and destabilise the limit cycle in comparison to the other grazing coefficients in the model. The critical value of mixotrophic grazing for the onset of chaotic dynamics, $\phi_{A P} \approx 0.0375$, is much less than the magnitude of the krill grazing on ice algae $\left(\phi_{A K}=0.50\right)$ or phytoplankton $\left(\phi_{P K}=0.25\right)$, or the magnitude of the salps grazing on phytoplankton $\left(\phi_{P S}=0.33\right)$. This suggests that the system is very sensitive to the inclusion of mixotrophy even in its simplest implementation of specialist grazing, with very small mixotrophic interaction being sufficient to destabilise a previously stable limit cycle. 


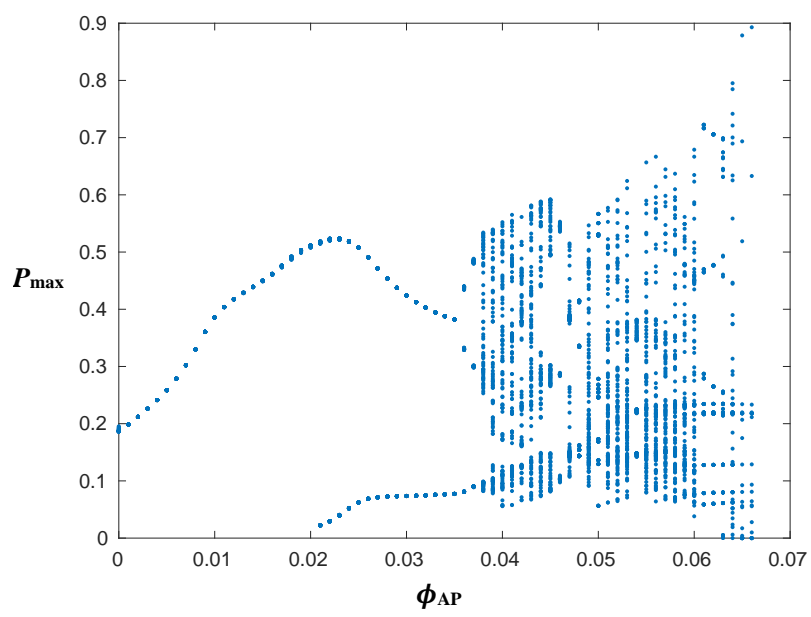

Fig. 5 The bifurcation transition diagram for the specialist mixotroph $P$ as $\phi_{A P}$ is increased, in terms of the maxima of $P$, for the SOEM system. Note: in this and all bifurcation diagrams we show, only the maximum $P$ value from each cycle is plotted. A single point on any vertical line indicates a repeating period-one cycle, two points indicate a period-two cycle, and so on until many points suggest chaos. Steady state solutions are not plotted, so the absence of points on any vertical line indicates either the system has a steady state solution or the population is extinct. For example, the period-one limit cycle shown in Fig. 3 is shown in this figure by the single dot at $P_{\max }=0.2$ on the $P_{\max }$ axis.

We now consider the effect on the system of allowing $P$ to function as a generalist mixotroph grazer on inorganic nutrient $N$ and ice algae $A$ by increasing $\phi_{A P}$ to non-zero values while this time setting $\rho=1$. The effect of increasing $\phi_{A P}$ is somewhat different in the generalist to the specialist version. We see from Fig. 6 that significant change occurs almost instantaneously in the system, and the onset of chaotic dynamics occurs at much smaller values of $\phi_{A P}$ than in the specialist case. The dramatic change in dynamics that occurs for any non-zero value of $\phi_{A P}$ is due to the generalist grazing formulation affecting the term describing the phytoplankton uptake of inorganic nutrient. This is sufficient in this example to prevent $P$ from growing, sending it and $S$ to immediate extinction leaving a stable $\left\{A^{*}, K^{*}, F^{*}, W^{*}\right\}$ system.

As the magnitude of $\phi_{A P}$ increases, the additional grazing is eventually sufficient to overcome the initial reduction in growth on inorganic nutrient, and the $P$ population reappears at $\phi_{A P} \approx 0.0125$ bringing with it the $S$ population in a high-period limit cycle. However, as $\phi_{A P}$ increases further, the $W, K$, and then $A$ populations go to extinction, so when $\phi_{A P}=0.084$ only a stable $\left\{P^{*}, S^{*}, F^{*}\right\}$ steady state remains. Again, this analysis reveals the sensitivity of the system to the inclusion of mixotrophy at the lowest trophic level, and the additional issues that the inclusion of low levels of generalist mixotrophic grazing can have in reducing existing grazing levels on other food sources. This suggests that some consideration may need to be given to the precise form that is used to represent generalist grazing for mixotrophs that 
may change their grazing preferences in response to external factors such as temperature [54].

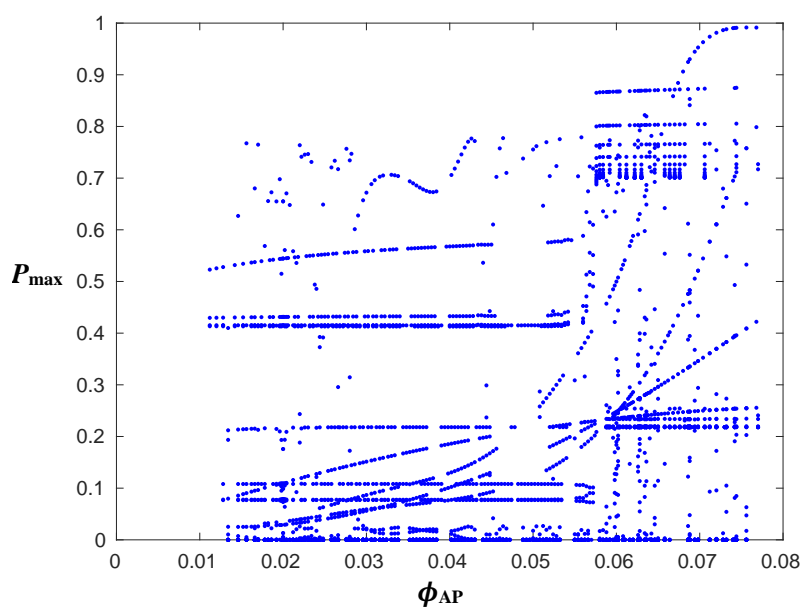

Fig. 6 The bifurcation transition diagram for $P_{\max }$ when $\rho=1$ (generalist mixotroph) as $\phi_{A P}$ varies for the SOEM system. Note $P$ is extinct for $\phi_{A P}<0.012$. Details as for Fig. 5. The horizontal and exponential lines of dots evident in the plot show patterns of regular behaviour occasionally emerging from the chaos.

To emphasise the sensitivity of the SOEM system to low levels of generalist mixotrophy, we include a bifurcation transition diagram for $A_{\max }$ (Fig. 7). We find a different complexity in this diagram, with different patterns of dots representing the different dynamics that are occurring in the different populations. However, regions of very complicated, probably chaotic dynamics are interspersed with regions with simpler, often period-one or -two limit cycle dynamics.

The difference in the effect of introducing mixotrophy in specialist or generalist form to the SOEM is due to the generalist grazing functional form requiring $A$ to be included into the function describing $P$ 's photosynthesis $\left(\mu_{P} R_{P} N\right) /\left(N+\rho A+\kappa_{P}\right)$ (equation (8)). When $\phi_{A P}=0, \rho$ must also equal zero as $P$ is not functioning as a mixotroph, and when $\phi_{A P}>0, \rho$ must be non-zero, and the denominator of the $P$ photosynthesis term changes abruptly from $\left(N+\kappa_{P}\right) \rightarrow\left(N+A+\kappa_{P}\right)$. The effect of including low levels of mixotrophy (small values of $\phi_{A P}$ ) can be, depending on the size of $A$ at the time, to lower substantially the rate of photosynthesis, and consequently the overall growth, of $P$. This is demonstrated in Fig. 8, where the growth functions in equation (8) are plotted. Here the addition of low levels of specialist mixotrophy barely affects the overall growth surface of $P$, which remains almost identical to the pure autotroph form. However, when small amounts of generalist mixotrophy are added, $P$ 's overall growth surface changes substantially. In the case of Fig. 


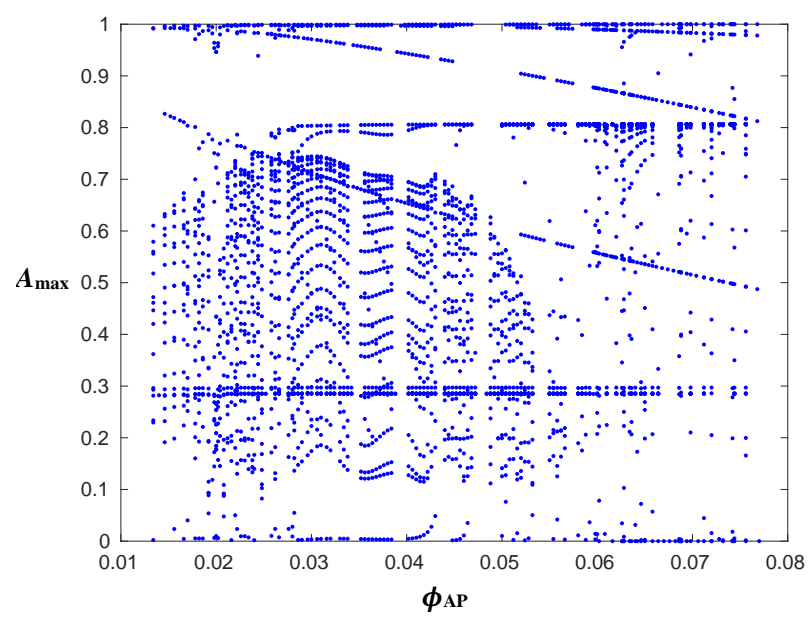

Fig. 7 The bifurcation transition diagram for $A_{\max }$ when $\rho=1$ as $\phi_{A P}$ varies for the SOEM system. Note the system has an $A K F W$ steady state for $\phi_{A P}<0.0125$, so $A_{\max }$ is constant and non-zero in this region. Details as for Fig. 6. Note patterns of regular behaviour embedded in the chaos as in Fig. 6.

6 , this is sufficient to change the sign of $P$ 's boundary eigenvalue, causing it to go extinct in simulations. This remains the case for $0 \leq \phi_{A P} \leq 0.0125$, after which the additional growth from mixotrophy exceeds the photosynthesis growth lost, $P$ 's boundary eigenvalue becomes positive, and for further increases of $\phi_{A P}$ the $P$ population can once again grow.
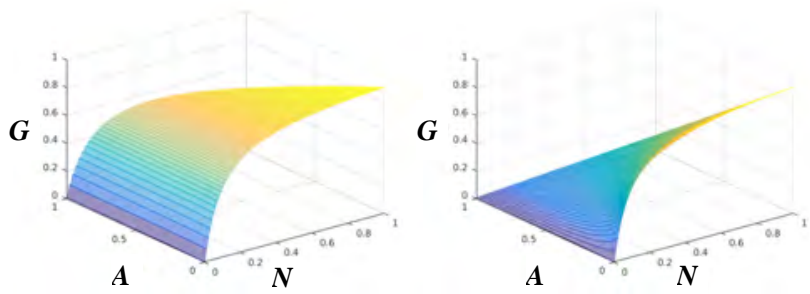

Fig. 8 The specialist (left) and generalist (right) mixotroph grazing surfaces for $P$ when $\phi_{A P}=0.01$ for the grazing functions (8). The generalist form causes a reduction in photosynthesis that does not occur in the specialist form, evident by the differences in the curves for large values of $A$. The photosynthesis function for $P$ without mixotrophy is indistinguishable from the specialist case. Note that due to the mass constancy condition, the grazing functions are not valid for $A+N>1$. 


$$
\begin{array}{ll}
G=\frac{\mu_{P} R_{P} N}{N+\kappa_{A P}}+\frac{\phi_{A P} A}{A+\kappa_{A P}}, & \text { for specialist mixotrophy, } \\
G=\frac{\mu_{P} R_{P} N+\phi_{A P} A}{N+A+\kappa_{A P}}, & \text { for generalist mixotrophy. }
\end{array}
$$

This limits the usefulness of the generalist grazing formulation when modelling "green" mixotrophs [6] that mostly photosynthesise and supplement this food source with only minor grazing. The SOEM system in its Table 1 parameterisation (Figs. 5 and 6 ) provides an example of when the choice makes a profound difference. Choosing to implement small levels of generalist mixotrophy unintentionally reduces $P$ 's photosynthesis and results in losses exceeding growth. $P$ cannot grow under these circumstances, and goes extinct, in this case taking $S$ with it. The zone in Fig. 6 where $P$ is extinct is entirely an undesirable artefact of the generalist grazing functional form, suggesting that the usual generalist forms are not a robust option for modelling mixotroph systems with low levels of mixotrophic grazing.

\subsection{Transition from Specialist to Generalist}

Although the ecological interpretation of the intermediate value $0<\rho<1$ is not clear, these intermediate values are mathematically valid, and we can investigate this region of parameter space to provide some support to the insights above. The bifurcation diagram for $P_{\max }$ for this parameter variation, with $\phi_{A P}=0.05$, a value that produced chaotic dynamics in all six populations in both the specialist and generalist cases, is shown in Fig. 9. An interesting result in Fig. 9 is the appearance of a period three limit cycle at $\rho \approx 0.05$, which is maintained to $\rho \approx 0.225$, at which point the dynamics become chaotic again. This chaotic regime is maintained until $\rho \approx 0.5$, after which the system appears to fall into a regime known as "pinball dynamics", a phenomenon observed in many fields, where "breather" populations spend some time at exponentially small levels before blooming vigorously, sometimes to dominate the entire system $[21,9]$. It appears that all populations remain extant in this regime, although if any population is examined over a specific period, it may remain at exponentially small levels that suggest it would not recover. With sufficiently accurate ode integrators, these populations in fact exhibit effectively "unpredictable" periods of explosive growth that poor ode integrators and pde integration schemes cannot reproduce. Similarly, solution codes that enforce artificial "minimum population levels" to ensure the survival of populations in systems with many populations will predict very different population dynamics to models with robust integrators. 


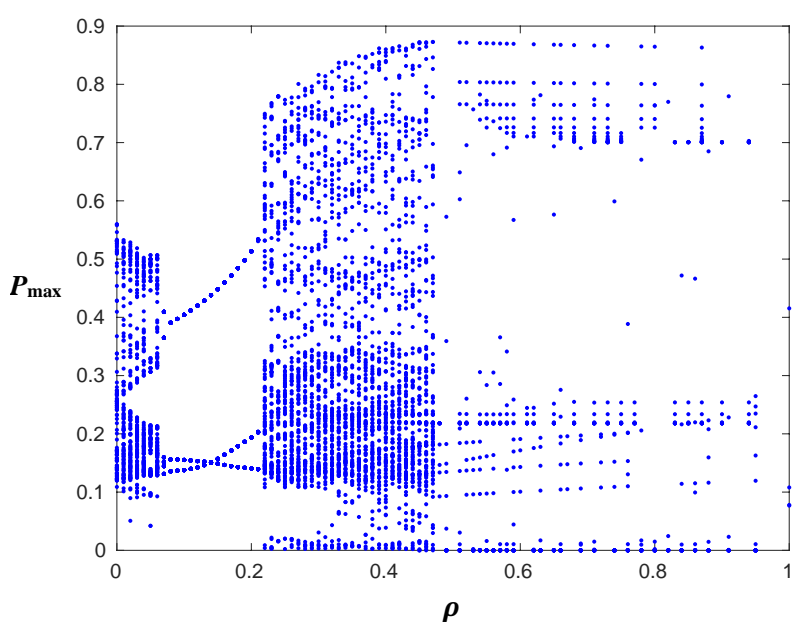

Fig. 9 The bifurcation transition diagram for $P_{\max }$ (the maximum value of the phytoplankton population over its irregular bloom cycles) when $\phi_{A P}=0.05$ (i.e. very weak mixotrophic grazing) as $\rho$ is increased from specialist $(\rho=0)$ to generalist $(\rho=1)$ grazing forms for the SOEM. Note the system alternates between regions of chaotic dynamics (dense dots) and regions of simple limit cycles (for example, the region $0.05<\rho<0.2$ ).

\subsection{Bifurcations in the Forced System}

We now consider the forced system, where we replace $R_{A}=1, R_{P}=1$, and $R_{W}=1$ used so far in equations 1 with the time varying forcings described by equations (3) - (6). We see from Fig. 10 that chaos is entrained into the system much earlier than in the unforced case, which maintained stable limit cycle dynamics over the range $0 \leq \phi_{A P} \leq 0.037$ (Fig. 5). In the forced case, fully chaotic dynamics are evident in the forced system by $\phi_{A P} \approx 0.007$, and are mostly maintained until $\phi_{A P}=0.116$. However, Fig. 10 also reveals that the forced system has windows of stability, with a multi-period limit cycle evident in the region $0.03 \leq \phi_{A P} \leq 0.04$, a single-period limit cycle evident in the region $0.068 \leq \phi_{A P} \leq 0.089$, and a robust period-four limit cycle forming and persisting for $\phi_{A P}>0.116$.

The effect of varying the magnitude of mixotrophic grazing on the forced system with generalist mixotrophy (Fig. 11) is interesting in that it appears that the forcing protects $P$ and $S$ from going extinct in the region $0<\phi_{A P}<$ 0.0125 as they did in the unforced version (Fig. 5), and extends the zone of chaotic dynamics significantly, with chaos now observed throughout the region $0.005<\phi_{A P}<0.158$. Although some structure is evident in the chaotic region (up to $\phi_{A P} \approx 0.158$ in Fig. 11 ) the system is generally chaotic throughout this region, apparently without any regions of stable dynamics.

Not evident in Fig. 11 is that $W \rightarrow 0$ when $\phi_{A P} \approx 0.106$, followed by $K \rightarrow 0$ when $\phi_{A P} \approx 0.158$. For $\phi_{A P}>0.158$ the system recovers a large amplitude limit cycle, this time in only $A, P, S, F$, which is maintained until 


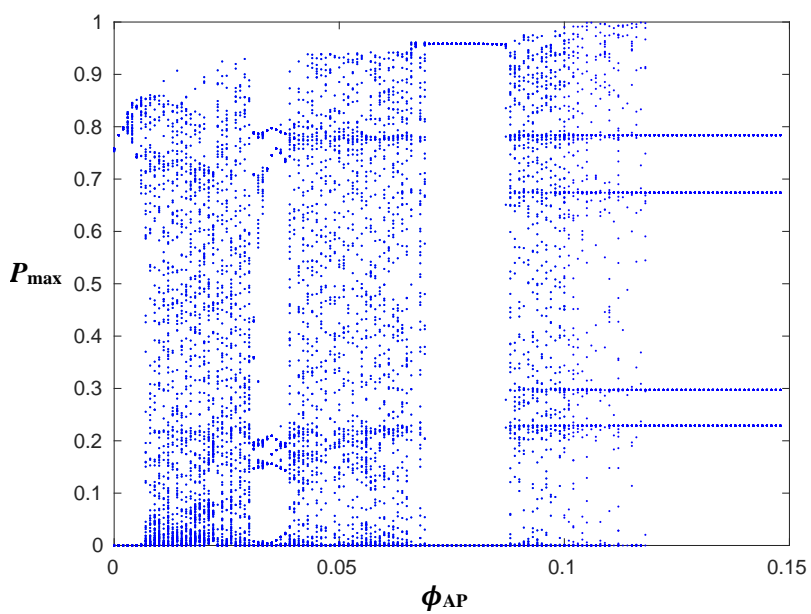

Fig. 10 The bifurcation transition diagram for the forced specialist mixotroph SOEM system, when $\rho=0$ and $\phi_{A P}$ is increased. Note the parameter windows of simple, regular period-one and period-four plankton blooms are interspersed, and in some cases intermixed, with generally irregular bloom responses.

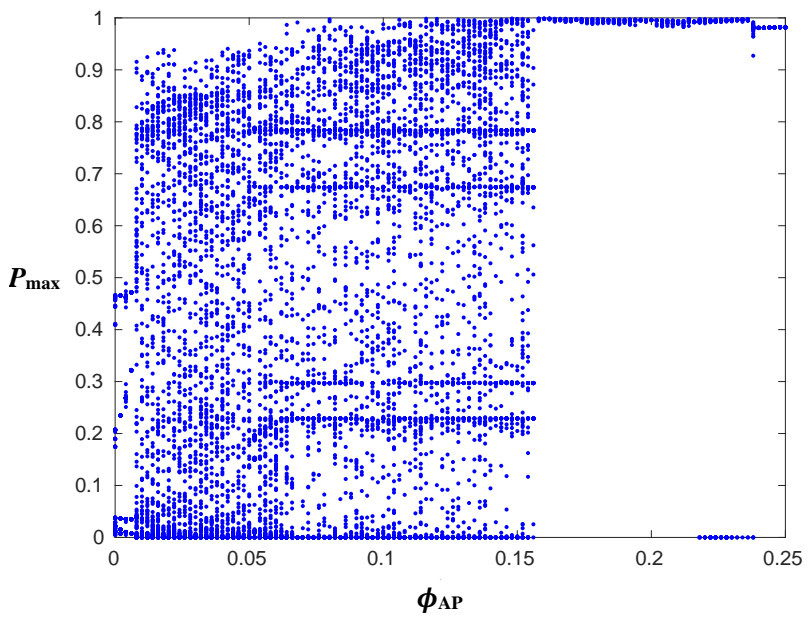

Fig. 11 The bifurcation transition diagram of the maximum value of $P\left(P_{\max }\right)$ for the forced generalist mixotroph SOEM, when $\rho=1$ and $\phi_{A P}$ is increased. The system is chaotic for all values of $\phi_{A P}>0$ until the system collapses at $\phi_{A P} \approx 0.16$ and $P$ settles into a limit cycle that has its maximum value near 1 .

$\phi_{A P}=0.25$. This is the largest value of $\phi_{A P}$ for which we retain a semblance of the original system, but it is still small compared to the phytoplankton and ice algae photosynthesis rates. However, it is of similar order to the rates of 
grazing of krill and salps on phytoplankton, and half the rate of krill grazing on ice algae.

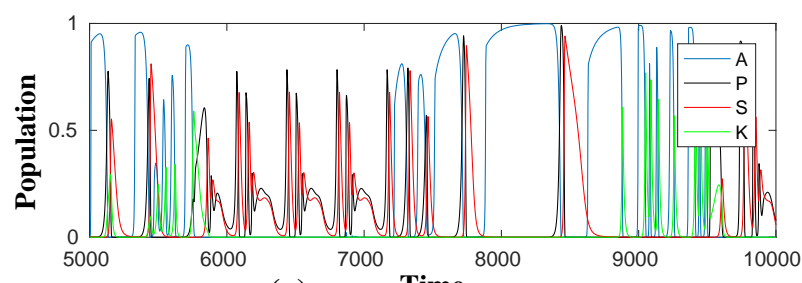

(a)

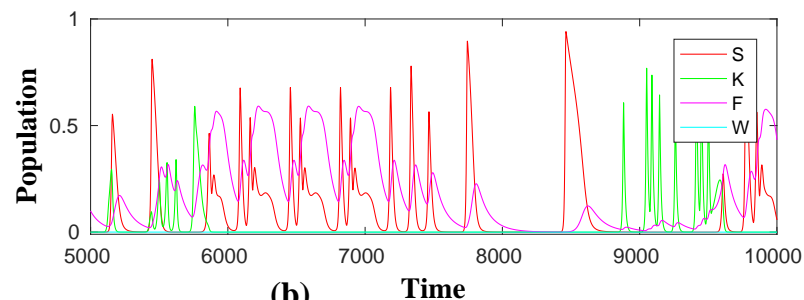

(b)

Fig. 12 A section of the time series for (a) the lower trophic levels, (b) the higher trophic levels of the forced specialist mixotroph SOEM, when $\rho=0$ and $\phi_{A P}=0.1$. Note the apparent extinctions of some populations for extended periods (for example, whales disappear for about a decade) before recovering at unpredictable intervals to dominate the system. The cycles are again irregular, despite the regular environmental forcings, indicating that endogenous cycling due to population interactions is at least as important as exogenous forcing due to a seasonally changing environment.

Figure 12 shows a section of the time series for the forced specialist SOEM food web when $\phi_{A P}=0.1$, revealing that under forcing, instead of $A, K, W$ going extinct, as occurred in the unforced case (Fig. 5), they survive and the dynamics become chaotic. The system also "decouples", with two "vertical" trophic groups of $(A, K, W)$ and $(P, S, F)$ emerging (see Fig. 1). These groups now alternate, each group dominating the system in turn (i.e. when $A, K, W$ bloom $P, S, F$ are small and vice versa). Further, the $W$ population varies between $3.3574 \times 10^{-9}$ and 0.0529 , exhibiting "breather" behaviour, where it remains at low (perhaps barely observable) levels for some time and then rapidly blooms..

Figure 13 shows the corresponding scenario for the forced generalist food web. Unlike the unforced case, when $\phi_{A P}=0.1$ and $\rho=1$, we no longer have a steady $(P, S, F)$ state (Fig. 6), but instead a chaotic oscillation involving all six populations. 

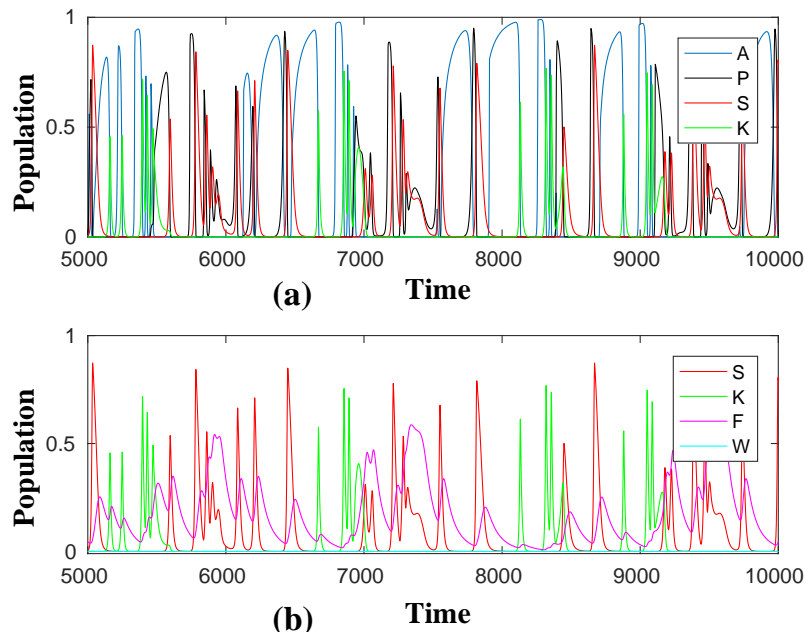

Fig. 13 A section of the time series for (a) the lower trophic levels, (b) the higher trophic levels of the forced generalist mixotroph $(A, P, S, K, F, W)$ system, when $\rho=1$ and $\phi_{A P}=0.1$. Note that the population cycles are not regular, despite the strong, regular environmental forcings.

\section{Discussion}

This study shows that mixotrophy matters, and in particular, that even subtle mixotrophic interactions at the lowest trophic level, can have significant implications for the dynamics of the whole ecosystem. Specifically, we first note that:

- the generalist grazing form, commonly used in plankton models where a predator has more than one prey, is inappropriate for mixotrophic phytoplankton, especially at small levels of mixotrophy, and

- the CN framework helps us to understand the reasons why the addition of small levels of mixotrophy counter-intuitively causes the mixotroph to go extinct initially, but later recover when mixotrophic grazing is sufficiently strong.

These results suggest models should include some mixotrophy, perhaps at varying rates related to climate change [54], but it should be introduced in a blended specialist/generalist grazer form. This is especially important if inspection of the value of the mixotroph's per capita growth function is only weakly positive at any of the boundary equilibrium points of the system.

The addition of mixotrophy in the standard generalist form results in the inclusion of a prey population term in the denominator of the mixotroph's photosynthesis term, which then causes a reduction in photosynthesis, and a reduction in overall growth when mixotrophic grazing is low. Low amounts of mixotrophy did not affect the phytoplankton population adversely when the 
specialist mixotrophy form was used. However, mixotrophy led to the extinction of the phytoplankton population when it initiated generalist mixotrophic grazing on ice algae in addition to photosynthesis. This counter-intuitive result occurred for all low values of generalist mixotrophy, until $\phi_{A P} \approx 0.0125$, at which point the gain from grazing was sufficient to offset the loss in photosynthesis.

Observations of Southern Ocean ecosystems show variability, often attributed to physical/climatic conditions of local sea ice extent, water temperature or nutrient supply. However, our analysis reveals that:

- chaotic solutions occur widely in parameter space, so that varying parameters to avoid this solution behaviour is usually not a successful option;

- further, adding seasonal forcing to the plankton and algae growth terms nearly always leaves the chaotic behaviour dominating, despite the relatively large magnitude of such forcings;

- these results suggest that it may be the spatial mixing (and/or the numerical schemes required to resolve it) that produce the common result of seasonal forcings suppressing chaotic dynamics in coupled ocean-ecosystem models.

The regular cycling of solar irradiance, sea ice extent, mixed layer depth, and whale grazing of krill allows irregular fluctuations of the bloom cycle, suggesting that anomalous krill years in Southern Ocean ecosystems may not necessarily be the result of physical processes, but may merely be a realisation of the endogenous dynamics of the population interactions.

The implications of these results for real world Southern Ocean ecosystems are subject to a significant caveat that arises from the formulation of the mixotrophic grazing terms $\mu_{P} R_{P} N P /\left(\rho A+N+\kappa_{A P}\right)$ and $\phi_{A P} A P /(A+$ $\left.\rho N+\kappa_{A P}\right)$. This form has been used in previous studies to consider the implications of specialist or generalist feeding strategies in omnivores [39], but the veracity of its extension to mixotrophs, where two fundamentally different types of metabolism are involved, is uncertain. The functional form we have used assumes trade-offs between the metabolic processes, where for example the commencement of heterotrophic feeding implies a reduction in autotrophic feeding. When $\rho=0$ (specialist mixotrophy), our formulations are consistent with Ward's [53] description of cellular level processes. While it is not certain that the formulation retains its verisimilitude when $\rho=1$, it remains a useful first approximation, and the formulation has a significant advantage that it facilitates a smooth transition between the two feeding strategies. Although the intermediate values of $\rho$ do not have clear ecological definitions they are mathematically valid and represent perhaps the most common scenario in the real world, that rarely are feeding strategies totally on or totally off, but commonly a blend. Alternative formulations might more realistically represent such scenarios, including the formulation:

$$
\rho\left(\frac{\theta \mu_{P} R_{P} N}{N+\kappa_{N P}}+\frac{(1-\theta) \phi_{A P} A}{A+\kappa_{A P}}\right) P,
$$


where $0<\theta<1$ determines the relative feeding fraction, suggested by a reviewer. We must bear in mind that functions that describe the feeding attributes of individuals are not necessarily the functions that describe the feeding attributes of their population $[15,16]$. The examination of the influence of alternative functions on the model dynamics and stability properties promises a productive future research theme.

Finally, we note the behaviour evident in some regions of the bifurcation analyses, that the maximum phytoplankton population sometimes remains at very low levels $(<0.1 \%)$ for long periods of time (years) before recovering quite reliably and stably to produce blooms that can dominate and use up the majority of the nutrient in the system. Such 'breather' blooms are common to a number of ecosystem models [9], particularly those with irregular outbreaks, and may be an attribute of Southern Ocean ecosystems. These widely separated, and essentially unpredictable, blooms make it difficult to determine experimentally when extinction, rather than a form of dormancy, has occurred.

\section{Conclusion}

A key outcome of this analysis is that ambiguity over trophic interactions at the very lowest levels of Southern Ocean food chains can lead to fundamental differences in the predictions of models that may be deployed to understand and predict critical issues ranging from climate change to the future of krill, whales, and other iconic Antarctic megafauna. The simple nutrient-phytoplankton-zooplankton (NPZ) model has been the workhorse of biological oceanography for the past forty years [14], but may need to be replaced by what is effectively a fundamentally different model, the nutrientphytoplankton-mixotroph-zooplankton (NPMZ) model, where the $\mathrm{P}$ and the $\mathrm{Z}$ represent smaller (perhaps $\sim 10 \%$ each) fractions of the plankton populations and the majority are mixotrophs $(\mathrm{M})$, in accordance with Flynn et al.'s estimates $[18,35]$.

The incorporation of mixotrophy is not a trivial change. A great advantage of the NPZ model, when written in the $\mathrm{CN}$ or similar framework, is that it reduces to two differential equations, for which the locations and stabilities of the equilibrium points that control the system dynamics may generally be obtained analytically. This attribute facilitates the use of these models as heuristics, and consequently two-population models have underpinned much of the development of theoretical ecology $[28,33,42]$. NPMZ models in contrast require three differential equations, and while the locations of equilibrium points may be available analytically, the stabilities of the equilibrium points where all three populations coexist are generally not available analytically, or if they are, are so algebraically complicated as to be of limited if any utility. While the competition and predation aspects of mixotrophy are relatively well understood in isolation, and Cropp and Norbury [6] have set out the basics of how these synthesise for mixotrophic population, the implications of these properties for the dynamical properties of NPMZ system are not straight-forward. How fun- 
damental changes in the dynamical properties of plankton ecosystems impact higher trophic level populations such as krill, whales, seals, and seabirds, is an order-of-magnitude more difficult problem.

Plankton are the foundation of all marine ecosystems. We rely on plankton to maintain oxygen in the atmosphere, to regulate climate, and to provide food for humans and the iconic marine megafauna that we cherish. That our assumptions of how plankton interact, that have underpinned our approaches to managing these natural resources, are missing a key component places in doubt the status quo of marine ecosystem science. We have demonstrated with a simple heuristic model that the inclusion of mixotrophy between plankton can produce substantially different model outcomes that are not constrained to the plankton, but flow through the entire ecosystem. Understanding the influence of plankton mixotrophy lies at the heart of the capability of ecosystem approaches to manage marine natural resources into the future.

Acknowledgements The authors thank the editors and two anonymous reviewers for their thoughtful and constructive contributions. JN, IMM and RC wish to thank Dr Keith Gillow for the cheerful and consummately competent provision of computing support at the Mathematical Institute, University of Oxford over a number of years. RC wishes to also thank the Mathematical Institute, University of Oxford; Lincoln College, Oxford; and St Hilda's College, Oxford for support in January 2018.

\section{References}

1. Atkinson, A., V. Siegel, E. Pakhomov, and P. Rothery. Long-term decline in krill stock and increase in salps within the Southern Ocean. Nature 432: 100-103. 2004.

2. Bates, M.L., S.M. Bengtson Nash, D.W. Hawker, J. Norbury, J.S. Stark, and R.A. Cropp Construction of a trophically complex near-shore Antarctic food web model using the Conservative-Normal framework with structural coexistence. J. Mar. Syst. 145, 1-14, 2015.

3. Boyd, I. L., Estimating food consumption of marine predators: Antarctic fur seals and macaroni penguins. J Appl Ecol 39: 103-119. 2002.

4. Clarke, A., and P.A. Tyler. Adult Antarctic Krill Feeding at Abyssal Depths. Curr Biol 18: 282-285. 2008.

5. Cropp, R.A., S.M. Bengtson Nash, and D. Hawker. A Model to Resolve the Dynamics of Organochlorine Pharmacokinetics in Migrating Humpback Whales. Environmental Toxicology and Chemistry. 33(7): 1638-1649. 2014.

6. Cropp, R.A. and J. Norbury. Population Interactions in Ecology: A Rule-Based Approach to Modeling Ecosystems in a Mass-Conserving Framework. SIAM REVIEW, 57(3): 437465. 2015.

7. Cropp, R.A., I.M. Moroz, and J. Norbury. Sequential boundary eigenvalue destabilisation (SeBEDes): An expert method for parameter screening and estimation in complex ecosystem models. Environmental Modelling and Software 90, 167-181, 2017.

8. Cropp, R.A., I.M. Moroz, and J. Norbury. The role of grazer predation strategies in the dynamics of consumer-resource based ecological models. Journal of Sea Research 125: 34-46. 2017.

9. Cropp, R.A. and J. Norbury. Simple predator-prey interactions control dynamics in a plankton food web model. Ecological Modelling, 20: 1552 -1565. 2009.

10. Cropp, R. A., and J. Norbury. The mechanisms of coexistence and competitive exclusion in complex plankton ecosystem models. Ecosystems 15: 200-212, 2012.

11. Cropp, R.A. and J. Norbury. Mixotrophy: the missing link in consumer-resource-based ecologies. Theoretical Ecology, 8: 245-260, 2015. 
12. Crane, K.W., and J.P. Grover. Coexistence of mixotrophs, autotrophs, and heterotrophs in planktonic microbial communities. Journal of Theoretical Biology, 262(3): 517-527. 2010.

13. Droop, M. R. Vitamin B12 and marine ecology. IV. The kinetics of uptake, growth and inhibition in Monochrysis lutheri. Journal of the Marine Biological Association UK 48:689-733. 1968.

14. Franks, P. NPZ Models of Plankton Dynamics: Their Construction, Coupling to Physics, and Application. Journal of Oceanography 58: 379-387, 2002.

15. Franks, P. Planktonic ecosystem models: perplexing parameterizations and a failure to fail. Journal of Plankton Research. 31:1299-1306, 2002.

16. Flynn, K.J. Modelling multi-nutrient interactions in phytoplankton, balancing simplicity and realism. Prog. Oceanogr., 56, 249-279, 2003.

17. Flynn, K.J., and A. Mitra. Building the "perfect beast": modelling mixotrophic plankton. Journal of Plankton Research 31(9): 965-992. 2009.

18. Flynn, K.J., D.K. Stoecker, A. Mitra, J.A. Raven, P.M. Glibert, P.J. Hansen, E. Graneli, and J.M. Burkholder. Misuse of the phytoplankton - zooplankton dichotomy: the need to assign organisms as mixotrophs within plankton functional types. J Plankton Res 35: 3-11. 2013.

19. Gentleman, W., A. Leising, B. Frost, S. Strom, and J.D. Murray. Functional responses for zooplankton feeding on multiple resources: a review of assumptions and biological dynamics. Deep Sea Research Part II: Topical Studies in Oceanography 50: 2847-2875. 2003

20. Grant, S.M., S.L. Hill, P.N. Trathan, and E.J. Murphy. Ecosystem services of the Southern Ocean: trade-offs in decision-making. Antarctic Science 25(5): 603-617. 2013.

21. Hadjiavgousti, D. and S. Ichtiaroglou. Allee Effect in Population Dynamics: Existence of Breather-like Behavior and Control of Chaos through Dispersal. I. J. Bifurcation and Chaos 16: 2001-2012. 2006.

22. Hill, S.L., E.J. Murphy, K. Reid, P.N. Trathan, and A. Constable. Modelling Southern Ocean ecosystems: krill, the food web, and the impacts of harvesting. Biol Rev 81: 581-608. 2006.

23. Holling, C. S., Some characteristics of simple types of predation and parasitism. Can Entomo 91: 385-398. 1959

24. Ichii, T., and H. Kato. Food and daily food consumption of southern minke whales in the Antarctic. Polar Biol 11: 479-487. 1991.

25. Ivlev, V. S., Experimental ecology of the feeding of fishes. Yale University Press, New Haven. 1961.

26. Jost, C., C.A. Lawrence, F. Campolongo, W. van de Bund, S. Hill, and D.L. DeAngelis. The effects of mixotrophy on the stability and dynamics of a simple planktonic food web model. Theoretical Population Biology 66: 37-51. 2004.

27. Koen-Alonso, M. A process-oriented approach to the multi-species functional response. In: Rooney, N. (Ed.), From Energetics to Ecosystems: The Dynamics and Structure of Ecological Systems. vol. 1. Springer, Netherlands, pp. 1-36. 2007.

28. Kot, M. Elements of Mathematical Ecology, Cambridge University Press, Cambridge, UK, 2001.

29. Lockyer, C., Growth and energy budgets of large baleen whales from the Southern Hemisphere., Mammals in the Seas, Vol. 3. FAO, Rome, pp. 379-487. 1981.

30. Loeb, V., V. Siegel, O. Holm-Hansen, R. Hewitt, W. Fraserk, W. Trivelpiece, and S. Trivelpiece. Effects of sea-ice extent and krill or salp dominance on the Antarctic food web. Nature 387: 897-900. 1997.

31. Lotka, A. J., Elements of physical biology. Wilkins and Kilkins, Baltimore. 1925.

32. May, R.M., J.R. Beddington, C.W. Clark, S.J. Holt, and R.M. Laws. Management of multispecies fisheries. Science 205: 267-277. 1979

33. May, R.M. Theoretical Ecology: Principles and Applications, Blackwell Science Inc, Princeton, Oxford, UK, 1981.

34. Michaelis, L. and M.L. Menten. Die Kinetik der Invertinwirkung. Biochem Z. 49: 333369. 1913.

35. Mitra, A., C. Castellani, W. Gentleman, S.H. Jonasdottir, K. Flynn, A. Bode, C. Halsband, P. Kuhn, P. Licandro, M.D. Agersted, A. Calbet, P.K. Lindeque, R. Koppelmann, 
E.F. Moller, A. Gislason, T.G. Nielsen, and M. St. John. Bridging the gap between marine biogeochemical and fisheries sciences; configuring the zooplankton link. Progress in Oceanography 129: 176-199. 2014.

36. Mitra, A., K.J. Flynn, J.M. Burkholder, T. Berge, A. Calbet, J.A. Raven, E. Graneli, P.M. Glibert, P.J. Hansen, D.K. Stoecker, F. Thingstad, U. Tillmann, S. Vage, S. Wilken, and M.V. Zubkov. The role of mixotrophic protists in the biological carbon pump. Biogeosciences 11: 995-1005. 2014.

37. Mitra, A., K.J. Flynn, U. Tillmann, J.A. Raven, D. Caron, D.K. Stoecker, F. Not, P.J. Hansen, G. Hallegraeff, R. Sanders, S. Wilken, G. McManus, M. Johnson, P. Pitta, S. Vage, T. Berge, A. Calbet, F. Thingstad, H.J. Jeong, J.A. Burkholder, P.M. Glibert, E. Graneli, and V. Lundgren. Defining planktonic protist functional groups on mechanisms for energy and nutrient acquisition: Incorporation of diverse mixotrophic strategies. Protist 167(2): 106-120. 2016.

38. Moeller, H.V., E. Peltomaa, M.D. Johnson, and M.G. Neubert. Acquired phototrophy stabilises coexistence and shapes intrinsic dynamics of an intraguild predator and its prey. Ecology Letters, 19(4): 393-402. 2016.

39. Moroz, I.M., R.A. Cropp, and J. Norbury. Chaos in plankton models: Foraging strategy and seasonal forcing. Ecological Modelling 332: 103-111. 2016.

40. Morozov, A. and S. Petrovskii. Feeding on Multiple Sources: Towards a Universal Parameterization of the Functional Response of a Generalist Predator Allowing for Switching. PLOS One 8(9): e74586: 1-8. 2013.

41. Murphy, E. J., R.D. Cavanagh, E.E. Hofmann, S.L. Hill, A. Constable, D.P. Costa M.H. Pinkerton, N.M. Johnston, P.N. Trathan, J.M. Klinck, D.A. Wolf-Gladrow, K.L. Daly, O. Maury, and S.C. Doney. Developing integrated models of Southern Ocean food webs: Including ecological complexity, accounting for uncertainty and the importance of scale. Progress in Oceanography 102: 74-92. 2012.

42. Pastor, J., Mathematical Ecology of Populations and Ecosystems, Wiley-Blackwell, Chichester, 2008.

43. Porter, K., Selective grazing and differential digestion of algae by zooplankton. Nature 244: 179-180.1973.

44. Sailley, S.F, M. Vogt, S.C. Doney, M.N. Aita, L. Bopp, E.T. Buitenhuis, T. Hashioka, I. Lima, C. Le Quere, and Y. Yamanaka. Comparing food web structures and dynamics across a suite of global marine ecosystem models. Ecological Modelling 261-262: 43-57. 2013.

45. Scheidat, M., H. Bornemann, E. Burkhardt, H. Flores, A. Friedlaender, K.H. Kock, L. Lehnert, J. van Franeker, and R. Williams. Antarctic sea ice habitat and minke whales. Annual Science Conference in Halifax, 22-26 September, Halifax, Canada. 2008.

46. Smith, E. L. Photosynthesis in relation to light and carbon dioxide. Proc. Natl. Acad. Sci. USA, 22: 504-511. 1936

47. Smout, S., C. Asseburg, J. Matthiopoulos, C. Fernandez, S. Redpath, S. Thirgood, and J. Harwood. The Functional Response of a Generalist Predator. PLoS ONE 5(5): 1-7. 2010.

48. D.K. Steinberg, K.E. Ruck, M.R. Gleiber, L.M. Garzio, J.S. Cope, K.S. Bernard, S.E. Stammerjohn, O.M.E. Schofield, L.B. Quetin, and R.M. Ross. Long-term (1993-2013) changes in macrozooplankton off the Western Antarctic Peninsula. Deep-SeaResearch I 101: 54-70. 2015.

49. Stitch, H.-B., and W. Lampert. Growth and reproduction of migrating and nonmigrating Daphnia species under simulated food and temperature conditions of diurnal vertical migration. Oecologia 61: 192-196. 1984

50. Vitousek, P.M., and P.A. Matson. Nutrient cycling and biogeochemistry. in S. Levin, editor. The Princeton Guide to Ecology. Princeton University Press, New Jersey, pp 330339. 2012.

51. Volterra, V., Variations and fluctuations of the numbers of individuals in animal species living together. In: Reprinted in 1931 in Chapman, R. N., (Ed.), Animal Ecology. McGraw Hill, New York. 1926.

52. Wang, S., M. Maltrud, S. Elliott, P. Cameron-Smith, and A. Jonko. Influence of dimethyl sulfide on the carbon cycle and biological production. Biogeochemistry 138:49-68. 2018. 
53. Ward, B.A., S. Dutkiewicz, A.D. Barton, A. D., and M.J. Follows. Biophysical aspects of resource acquisition and competition in algal mixotrophs. The American Naturalist, 178(1): 98-112. 2011.

54. Wilken, S., J. Huisman, S. Naus-Wiezer, and E. Van Donk. Mixotrophic organisms become more heterotrophic with rising temperature. Ecol. Lett. 16: 225-233. 2013. 